\title{
The Aberration Corrected JEM-2200FS at Brookhaven
}

\author{
Yimei Zhu, ${ }^{*}$ R. Klie, ${ }^{*}$ T. Tomita, ${ }^{* *}$ H. Sawada, ${ }^{* *}$ K. Nagahata, ${ }^{* *}$ \\ * Center for Functional Nanomaterials, Brookhaven National Laboratory, Upton, NY 11973 \\ ** JEOL Ltd., 3-1-2 Musashino, Akishima, Tokyo, Japan
}

The Brookhaven JEM-2200FS electron microscope has been installed recently. This JEOL instrument is a $200 \mathrm{kV}$ high-resolution field-mission TEM/STEM equipped with an in-column energy filter. When it is completed by the end of 2005, the instrument will have two CEOS Cs aberration correctors [1], one for the probe forming lens and the other for the objective lens [2], and a JEOL monochromator. Figure 1 shows the instrument at Brookhaven. To ensure the instrument will meet its specifications achieving sub- $\AA$ spatial resolution and sub-eV energy resolution, the microscope lab was carefully designed for the instrument to minimize mechanical vibration, acoustic noise, temperature variation and magnetic fields.

Currently only the probe corrector has been installed and its performance has been tested. Fig. 1 also shows an image of a focused beam with the probe Cs-corrector on. The line scan of the intensity distribution suggested the probe size is about $0.11 \mathrm{~nm}$ at the full width and half maximum with a beam current of eight times more than of the uncorrected probe. Spatial resolution of the STEM was evaluated using the annular dark-field (ADF) imaging mode. Fig.2 (a-b) shows the high-resolution images of the Si dumbbells in a $<110>$ orientation with the Cs corrector on $(\mathrm{Cs}=0.01 \mathrm{~mm}$, Fig. $2 \mathrm{a})$ and its corresponding power spectrum. Histogram analysis of Fig.2(a) indicates that the black level of the image contrast is not clipped (Fig.2(c)), thus, a probe-size of $1.05 \AA$ in all directions can be claimed since the (333) and the (511) spots are visible in Fig.2(b). Ronchigram from an amorphous germanium shows that the coherent convergence half-angle (flat phase field) can be increased from $14 \mathrm{mrad}$ to $40 \mathrm{mrad}$ when the corrector is used (Fig.2d-e). The spherical and chromatic aberration coefficients of the pre-field and post-field of objective lens of the original JEM2200FS were both 0.5 $\mathrm{mm}$. After correcting the main aberrations, Cs was measured to be less than $0.01 \mathrm{~mm}$ and the Cc was estimated to be $1.4 \mathrm{~mm}$. Other aberrations up to the third order can also be corrected (the aberration coefficients of second order coma and three-fold astigmatism were less than $0.1 \mu \mathrm{m}$ and the third order of star aberration and four-fold astigmatism were less than $3 \mu \mathrm{m})$. Fig.2(f-g) shows the calculated phase plate and a tableau with different beam tilts [3]. It was reported that with the objective lens corrector, the image resolution of the instrument in TEM mode can be improved from $0.19 \mathrm{~nm}$ to $0.12 \mathrm{~nm}[2]$. The EELS performance (measured energy resolution without the monochromator was $0.7 \mathrm{eV}$ ) using the Omega filter will be also presented [4].

\section{References}

[1] M.Haider, S.Uhlemann and J.Zach, Ultramicrosc., 81 (2000) 163

[2] H.Sawada, T.Tomita, et al, Microsc. Microanal. 10 (Suppl 2) CD976, CD1004 (2004).

[3] Parameter used in the calculations: defocus $C_{1}=2.369 \mathrm{~nm}, 2$-fold astigmastism $A_{1}=9.775 \mathrm{~nm}, 3$-fold astigmatism $\mathrm{A}_{2}=38.41 \mathrm{~nm}$, Axial coma $\mathrm{B}_{2}=64.59 \mathrm{~nm}$, spherical aberration $\mathrm{C}_{3}=4.784 \mathrm{um}$, 4-fold astigmatism $\mathrm{A}_{3}=2.887 \mu \mathrm{m}$, star aberration $\mathrm{S}_{3}=1.74 \mu \mathrm{m}$ and 5-fold astigmatism $\mathrm{A}_{4}=81.93 \mu \mathrm{m}$.

[4] Work supported by the US DOE, under Contract No. DE-AC02-98CH10886. 


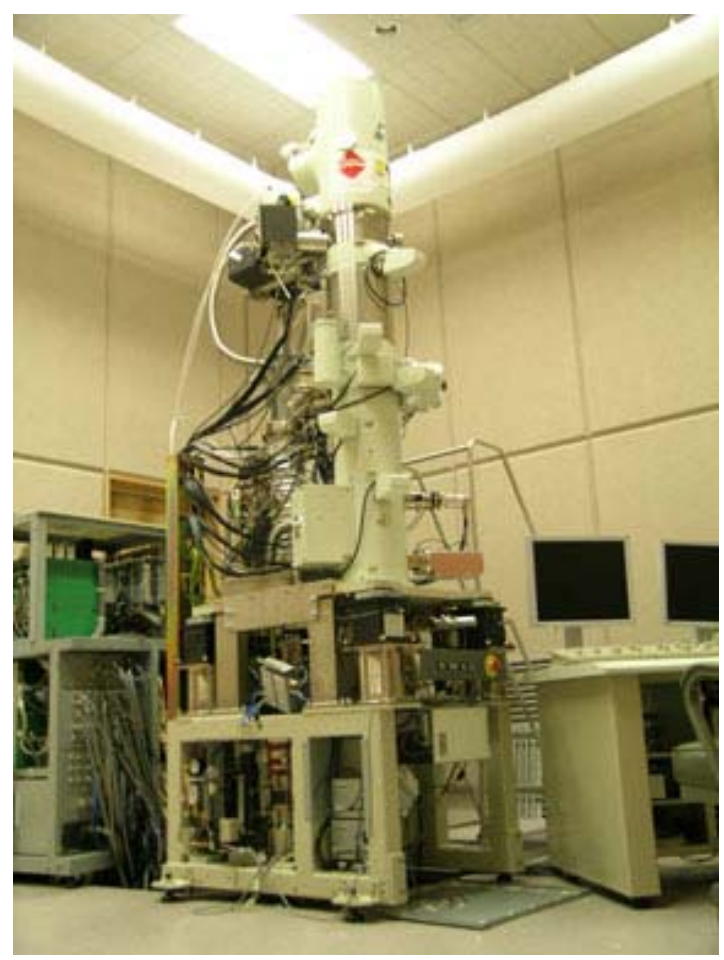

c
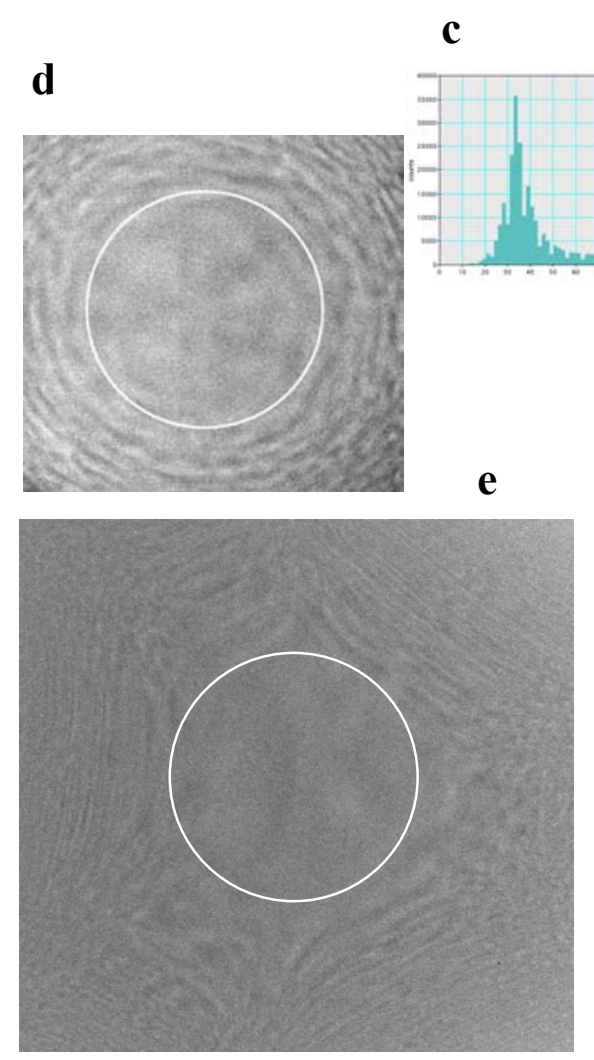

e

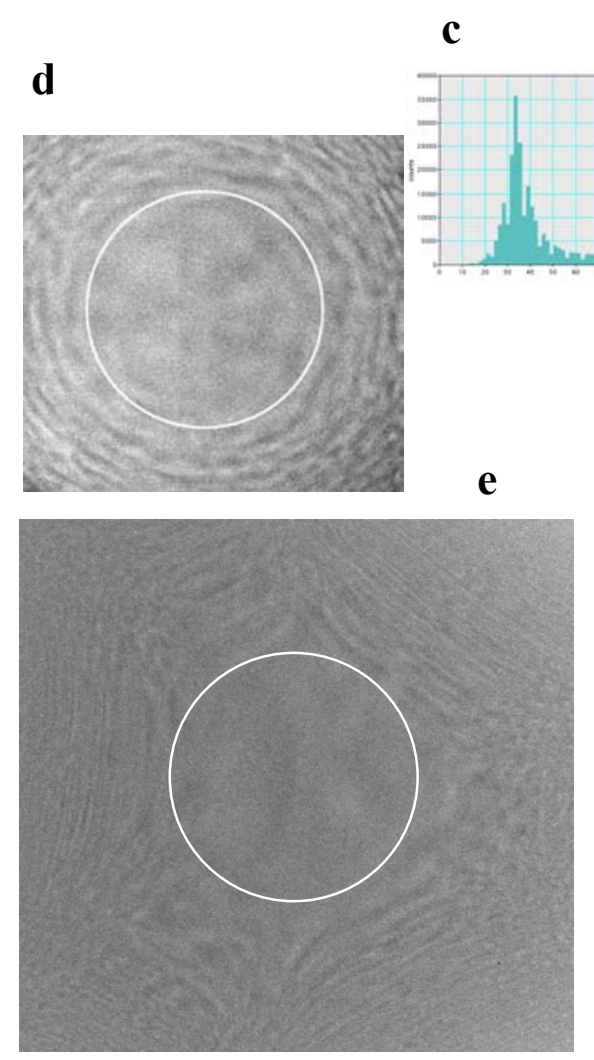

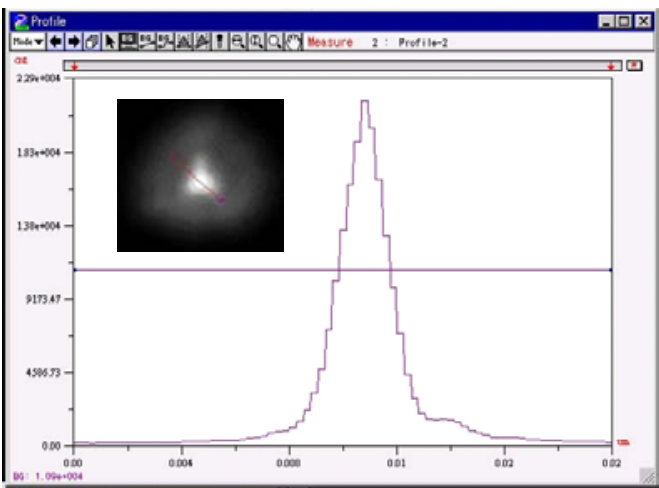

Fig.1 Left: The aberration corrected JEM-2200FS at Brookhaven. The microscope lab was carefully designed for the instrument with an isolated $1 \mathrm{~m}$-thick concrete slab, acoustic damping and a special air-flow system to minimize vibration, acoustic noise and temperature variations. Above: an image of the probe for scanning mode. The line-scan of the intensity distribution shows a full width of half maximum about $0.11 \mathrm{~nm}$. b

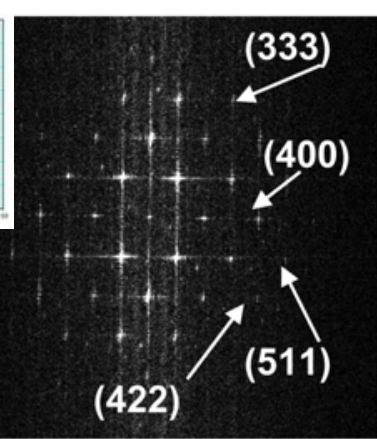

f

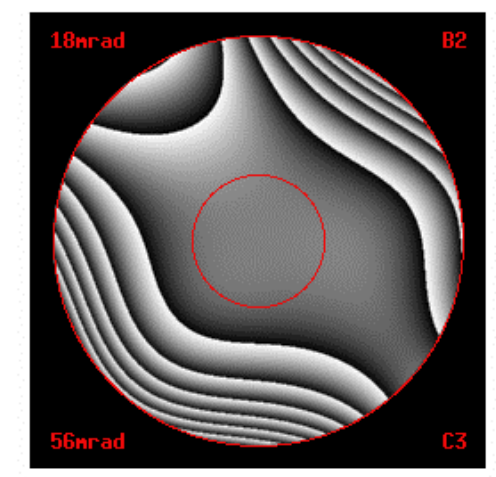

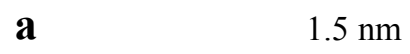

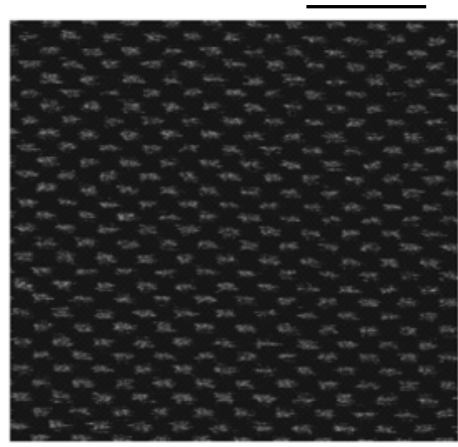

g

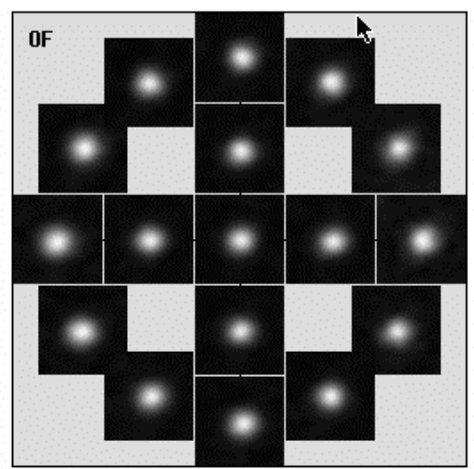

Fig.2 (a-b) A STEM ADF image and its corresponding diffractogram of Si (110) after aberration correction of the illumination system. (c) The histogram of (a) indicating the back-level of the image contrast is not clipped. (d-e) Ronchigrams of Ge showing increased coherent convergence half-angle before (d) and after (e) aberration correction. The circle in (d) and (e) corresponds to $14 \mathrm{mrad}$ and $40 \mathrm{mrad}$, respectively. (f-g) Calculated phase plate (the small and large circle corresponds to 18 and 56mrad, respectively) and a tableau of power spectra with various beam tilts. Parameters used are described in [3]. 